Relations industrielles

Industrial Relations

\title{
Pierre Elliott TRUDEAU : La grève de l'amiante. Montréal, Éditions du Jour, 1970, 430 pp.
}

\section{Jacques Gagnon}

Volume 41, numéro 3, 1986

URI : https://id.erudit.org/iderudit/050249ar

DOI : https://doi.org/10.7202/050249ar

Aller au sommaire du numéro

Éditeur(s)

Département des relations industrielles de l'Université Laval

ISSN

0034-379X (imprimé)

1703-8138 (numérique)

Découvrir la revue

Citer ce compte rendu

Gagnon, J. (1986). Compte rendu de [Pierre Elliott TRUDEAU : La grève de l'amiante. Montréal, Éditions du Jour, 1970, 430 pp.] Relations industrielles / Industrial Relations, 41(3), 669-670. https://doi.org/10.7202/050249ar

Tous droits réservés @ Département des relations industrielles de l'Université Laval, 1986
Ce document est protégé par la loi sur le droit d'auteur. L’utilisation des services d'Érudit (y compris la reproduction) est assujettie à sa politique d'utilisation que vous pouvez consulter en ligne.

https://apropos.erudit.org/fr/usagers/politique-dutilisation/ 
tangible rewards to the participant, for his/her extra effort and for the benefits accruing to the company?), sharing management level information with employees (is this information available and made clear to all interested persons?), guaranteed individual rights (are there guarantees to protect critical speech and organizing among the employees?), an independent appeals system (in case of reprisal or dispute, is there a trustworthy system for fair appeal?), and a complex participatory/democratic consciousness (are people able and willing to attach themselves to the common good and multiply it by joint effort?).

It is not easy to reconcile self-reliance with receptivity to others' needs, individualism with loyalty to others, activism with obedience to the common will, authority with participation, economic goals (profit) with social goals (community). «Democratization is unlikely to survive if only the managers understand democracy. They must be held to democratic practice by the managed». (p. 103)

The success of work democratization operates through a positive or reinforcing cycle against a negative or extinguishing cycle. The latter appears when the components of democratization (above mentioned) are not available and the employees face too many rejections of their attempts at participation (p. 112). The model offered by Bernstein is a developmental one. In the case of the lack of any one of six components mentioned above "either the firm must alter the system and adopt a new component - the relevant missing one(s) - to give the employees more rights and power - or the firm may abort the attempt at democratization because management is unwilling to accept any expansion of workers' power, or because the workers themselves do not feel capable of more responsibility and power» (p. 117).

Democratization at work needs to be cultivated and constantly reinforced in order to provide a genuine humanitarian content into the existing structures. «Democratization cannot function well until the workers themselves want it» (p. 117). Several variables are required, generating simultaneously within certain specified ranges in order to promote a genuine democratization. Involvement of workers in the collective participation in management in order to succeed needs to be a satisfactory answer to several existential problems of real importance and not just a doctrine of an abstract nature. Even the best will of reformers cannot assure success as long as a gap remains between intentions of the promotors and the expectations of the employees. The model proposed by Bernstein offers some useful suggestions to anybody involved in the process of work democratization.

Alexander J. MATEJKo

University of Alberta

\section{TROIS ÉTUDES DE GRÈVES COMPARÉES}

\section{INTRODUCTION}

Rares sont les monographies consacrées aux conflits de travail. Aussi n'y a-t-il que plus d'intérêt à s'attarder sur le peu qui a été publié au fil des ans. Ainsi voulons-nous revenir sur trois études consacrées à trois grèves célèbres au Québec: Asbestos 1949, Noranda 1953-54 et United Aircraft 1974-75.

Nous voulons aussi comparer les conflits entre eux et en relever les principales différences et ressemblances. Dans les trois cas, il s'agit de conflits très longs, impliquant des multinationa- 
les et des demandes de retenue de la cotisation syndicale à même le chèque de paie. À d'autres égards, ces conflits sont dissemblables: les secteurs d'activité économique sont différents, de même que les syndicats impliqués, ainsi que les périodes et régions où se situent ces grèves.

Les études elles-mêmes comportent plus de différences que de ressemblances. La grève de l'amiante est une oeuvre de collaboration impliquant une dizaine d'auteurs (journalistes, avocats, universitaires et syndicalistes). Analyse socio-économique d'une grève (Noranda 1953-54) est le travail d'un professeur des HEC de Montréal et La grève de la United Aircraft, celle d'un enseignant de science politique au CEGEP de Saint-Hyacinthe. Cela explique sans doute les différences de «format» des trois volumes: le premier comporte dix chapitres et 430 pages, le second a cinq chapitres et 218 pages et le troisième contient quatre chapitres et 115 pages. Une constante cependant unit les trois études: elles ont toutes été publiées longtemps après la grève qu'elles analysent...

La grève de l'amiante, en collaboration, sous la direction de Pierre Elliott Trudeau, Montréal, Éditions du Jour, 1970, 430 p. (1 ${ }^{\text {re }}$ éd. Cité Libre, 1956).

Il s'agit certainement de la plus connue des trois études que nous abordons et aussi de la seule à être rééditée. Sans doute profite-t-elle de la notoriété de ses auteurs qui ont fait leur bonhomme de chemin entre 1956 et 1970 . L'un est devenu Premier ministre du Canada, l'autre, Secrétaire d'État, etc. Mais c'est plutôt le contenu de l'ouvrage qui nous intéresse. Il est divisé, nous l'avons dit, en dix chapitres auxquels il faut ajouter un avant-propos, une préface, un épilogue et quatre appendices. Ce qui saute aux yeux, à prime abord, c'est qu'à peine la moitié du livre concerne directement la grève d'Asbestos. Les autres chapitres traitent plutôt de la province de Québec au moment de la grève, de l'histoire financière de l'industric de l'amiante, de l'histoire du syndicalisme dans cette même industrie et de la situation six ans après la grève. Les chapitres qui touchent spécifiquement la grève étudient son historique et celui des négociations puis certains acteurs impliqués dans le conflit: l'Église, l'administration publique, la presse et le mouvement syndical. Ce qu'il faut retenir de tout cela, c'est que les collaborateurs ont voulu d'abord situer le conflit dans son contexte socio-économique le plus large, pour ensuite détailler certains aspects plus importants de la grève elle-même. Néanmoins, les développements sont variables puisque le nombre de pages oscille entre 14 et 92 pages par chapitre, le chiffre moyen se situant autour de 33 pages.

Cependant, le talon d'Achille de ces études ne réside pas dans leurs dimensions mais dans leurs contradictions évidentes. Ainsi, on peut se surprendre que Trudeau écrive qu'il s'est agi là d'un «épisode-clé d'émancipation sociale qui a pu se dérouler sous l'impulsion de forces purement issues du monde industriel, sans déviation confessionnelle ni nationaliste» (p. 401). Pourtant, dans le même livre, Gérard Dion a rapporté de façon complète le rôle-clé joué par l'Église dans ce conflit (p. 239-262). Et Gérard Pelletier a décrit la position pro-syndicale de son propre journal, Le Devoir, et de l'ensemble de la presse catholique (p. 277-318). Quant à Maurice Sauvé, il nous dit en détail d'où proviennent les fonds d'appui aux grévistes (p. 319-344). Ce sont bel et bien des institutions québécoises, notamment catholiques, qui ont financé une grosse partie de la grève. Ainsi, les syndicats canadiens ne fournissent que $14000 \$$ alors que les diocèses du Québec collectent $167558 \$$ en plus de prêter $50000 \$$. Cela représente le tiers du coût de la grève évaluée à $600000 \$$.

Par ailleurs, dans un article de 1969, Hélène David fait une mise au point par rapport à cet "épisode-clé d'émancipation sociale» que fut la grève d'Asbestos, selon Trudeau. Elle s'étonne qu'on ait présenté la grève comme une si grande victoire ouvrière, devant le résultat concret des négociations. Les travailleurs ont tout juste réussi à sauver les meubles en obtenant au fil des 
ans l'augmentation des salaires et congés payés, et le congédiement des briseurs de grève. Par contre, ils n'ont jamais pu obtenir leurs revendications de départ. Il n'y a pas eu de cotisation syndicale obligatoire retenue à la source. Ni de consultation sur les promotions, transferts et congédiements. Ni le droit de refuser ou d'accepter individuellement les cadences de production. Le mot de la fin reste à Hélène David quị «propose que le conflit de l'amiante soit vu comme une situation où l'éclatement ouvert de la lutte de classes a engendré un conflit entre l'Église, défendant un modèle communautaire, et l'État, assurant l'équilibre de la société industrielle capitaliste. Ce modèle d'analyse permet, à partir des positions des acteurs - les ouvriers, les entreprises, l'État et l'Église - d'éclairer les contradictions dans leurs rapports réciproques et ainsi de rendre compte d'éléments non intégrés par les interprétations jusqu'ici proposées». («La grève et le Bon Dieu», in Sociologie et Sociétés, vol. 1, no 2, PUM, 1969.)

Analyse socio-économique d'une grève, par Jean Mehling, Montréal, HEC et Beauchemin, $1963,218 \mathrm{pp}$.

Comme son titre l'indique, le professeur Mehling veut lui aussi étudier un conflit de travail dans son contexte socio-économique le plus large. Mis à part les textes d'introduction et de conclusion, ses cinq chapitres portent respectivement sur la grève dans son contexte historique et conjoncturel; le rôle de la compagnie dans le développement des activités locales; sa capacité de résistance face à la grève; celle des travailleurs; et enfin, le coût de la grève et la capacité de résistance à long terme des parties en conflit. Notons que le traitement est plus équilibré que dans le volume précédent, puisque chaque chapitre compte de 24 à 50 pages ${ }^{1}$.

Véritable travail de bénédictin, cette étude ne cache ni ses lacunes, ni ses difficultés. Quatre ans après le conflit, l'auteur a épluché les dossiers des ministères fédéraux du Travail et des Mines. Il a aussi interrogé «des ouvriers réputés pour leur antipathie à l'égard du syndicat, des dirigeants syndicaux, des syndiqués réunis en groupe dans le local syndical, des religieux qui avaient pris part de diverses façons au conflit, des journalistes locaux, des hôteliers, des commerçants, des fonctionnaires, des hommes politiques, des employés de la Compagnie, etc.» (p. 51). Il a enfin mis à contribution les meilleures ressources comptables de l'École des Hautes Études commerciales. Malgré tout cela, il ne peut que conclure que «dans l'exemple de la grève de la Noranda Mines, le syndicat n'était pas en mesure, même au prix de longues recherches socio-économiques, de prendre une décision parfaitement rationnelle» (p. 214). L'auteur en prend pour preuve sa propre analyse du conflit qu'il juge trop incomplète.

Cependant, tout n'est pas négatif dans l'étude du professeur Mehling, tant s'en faut. L'auteur réussit à nous brosser le portrait fidèle d'une communauté isolée où une compagnie très puissante fait face à une force ouvrière organisée mais fragile. En effet, les travailleurs de la Noranda n'ont ni épargne ni biens de fonds pour soutenir leur grève, et ils doivent compter exclusivement sur les secours de leur centrale syndicale. Le principal enjeu du conflit est moins la hausse salariale que la "clause de retenues syndicales" et bien sûr, la capacité future de négociation des deux parties. Le conflit se termine par une victoire non équivoque de la partie patronale, après la cessation des paiements de secours de la centrale syndicale américaine. Contrairement au cas d'Asbestos, il y a eu peu d'intervention extérieure, que ce soit de l'Église, de l'État ou de l'opinion publique.

1 Il est à noter que le volume de Trudeau et al. fait allusion au conflit de RouynNoranda: «À cinq ans de distance, les mineurs de l'Abitibi étaient animés du même esprit qui avait animé ceux d'Asbestos et de Thetford-Mines. Ils retinrent même, pour la durée de leur grève, les services de M. René Rocque, ancien organisateur de la C.T.C.C., dont le nom est indissolublement lié aux événements de 1949» (Réginald Boisvert, in Trudeau, op. cit., p. 365). 